\title{
Effectiveness of an Internet-Based Self-Help Intervention versus Public Mental Health Advice to Reduce Worry during the COVID-19 Pandemic: A Pragmatic, Parallel-Group, Randomized Controlled Trial
}

\author{
Hanna Heckendorf Dirk Lehr Leif Boß \\ Department of Health Psychology and Applied Biological Psychology, Institute of Psychology, Leuphana University \\ of Lüneburg, Lüneburg, Germany
}

\section{Keywords}

COVID-19 · Worry · Internet-based intervention · Self-help · Randomized controlled trial

\begin{abstract}
Introduction: The mental health burden for the general population due to the COVID-19 pandemic has been highlighted. Evidence on effective, easily accessible public health interventions to reduce worry, a major transdiagnostic riskfactor for, e.g., anxiety and depression, is scarce. Objective: In a pragmatic randomized controlled trial, we aimed to assess whether an internet cognitive-behavioral self-help intervention could reduce worry more than public mental health advice in the general population. Methods: Eligible internet users above the age of 18 were recruited from the German general population and randomly assigned, to either get.calm-move.on (GCMO), a 10-day unguided, internet-based self-help intervention, or mental health advice waiting group (MHA-W, receiving officially endorsed mental health recommendations). The primary outcome was level of worry, using the Penn State Worry Questionnaire (PSWQ), 2 weeks after randomization. Baseline assessment and
\end{abstract}

karger@karger.com www.karger.com/pps

Karger"

BOPEN ACCESS
(C) 2022 The Author(s)

Published by S. Karger AG, Basel

This is an Open Access article licensed under the Creative Commons Attribution-NonCommercial-4.0 International License (CC BY-NC) (http://www.karger.com/Services/OpenAccessLicense), applicable to the online version of the article only. Usage and distribution for commercial purposes requires written permission. 2-month and 6-month follow-ups were conducted. The trial was registered at the German Clinical Trials Registry (DRKS00021153). Results: Between April 7, 2020 and December 11, 2020, we randomly assigned 351 individuals to receive either GCMO $(n=175)$ or MHA-W $(n=176)$. Participants receiving $\mathrm{GCMO}(\mathrm{PSWQ}=46.6$; change -10.3$)$ reported significantly less worrying at post-intervention $\left(F_{1,219}=12.9 ; p\right.$ $<0.001 ; d=0.38$ ) than MHA-W controls (PSWQ $=51.6$; change -5.1). Improvements were also seen on most secondary outcomes, including symptoms of anxiety and depression, general well-being, resiliency, and emotion regulation skills. Improvements made from baseline were stable until the 6-month follow-up. Conclusions: This internet-based selfhelp intervention providing cognitive-behavioral techniques to cope with the threatening pandemic situation is effective in reducing worry in the general population and should complement existing and potentially effective mental health recommendations.

(c) 2022 The Author(s)

Published by S. Karger AG, Basel

Hanna Heckendorf and Dirk Lehr contributed equally and share first authorship.
Correspondence to:

Dirk Lehr, lehr@leuphana.de 


\section{Introduction}

Societies worldwide have felt threatened by the SARSCoV-2 virus pandemic, with COVID-19 morbidity and mortality being the most salient health consequences. Studies on prior virus outbreaks highlight the adverse influence of pandemics on mental health as well [1]. There is evidence, from several observational studies, that similar negative effects on mental health should be expected with the current pandemic [2-5]: a "quiet" consequence of the pandemic. However, evidence from prior epidemics or pandemics from well-designed interventional studies on successful strategies to prevent adverse mental health outcomes is limited [6].

From a psychological perspective, pandemics can be characterized as highly volatile, mainly controlled by external factors with an uncertain future, in which the potential for several negative outcomes exists. In general, such characteristics are likely to elicit worry, symptoms of anxiety, and depression. This can be exacerbated by self-isolation and quarantine measures, physical distancing, and working remotely. COVID-related medical worries include the fear of infection, fear of the healthcare system being overloaded, and fear that either oneself or close others who contract COVID-19 syndrome will have a severe, chronic, or potentially fatal COVID-19 course [7-9]. Non-medical worries include negative repetitive thoughts about one's general life situation, as well as psychosocial and socioeconomic consequences of the crisis [7-9].

Worrying as a form of future-oriented negative thinking is characterized by repetitive thoughts and images that are negative affect-laden, perceived as difficult to disengage from, and focused on negative content $[10,11]$. Negative repetitive thoughts about the future are a transdiagnostic risk factor for the development and maintenance of depression and anxiety disorders [12], and partly explain their comorbidity [13]. Therefore, interventions targeting worrying might reduce psychological distress and positively affect symptoms of depression and anxiety. Moreover, transdiagnostic interventions are one way to reach the broader population [14].

From a public mental health perspective, it is important to reach the broader population with low-threshold, easily accessible interventions that can be delivered on a large scale and by keeping physical distance. Accordingly, early in the pandemic, the World Health Organization (WHO) published educational material for mental health protection on the internet, including advice for individuals to, for example, limit worry and focus on self-efficacy in the face of adversity [15]. There is meta-analytic evidence supporting the premise that psychoeducation is effective at reducing psychological distress and depression [16]. Internet-based self-help interventions also have the potential to reach a large group of distressed individuals. Other meta-analytic evidence suggests that internetbased self-help interventions reduce distress both in the general population [17] and in subclinical settings [18]. As such, this approach has the potential to provide help for the general population to cope with worries in the pandemic [19]. However, in these meta-analyses a high degree of heterogeneity was identified, revealing beneficial effects for only some interventions [17].

For the current pandemic, Wahlund et al. developed a self-help internet intervention targeting medical and non-medical COVID-19 worries [20]. In a population with excessive COVID-19-related worries and already existent losses in psychosocial function, these investigators detected greater reductions in the symptoms of generalized anxiety disorder (GAD) in the intervention as compared to a non-active waitlist control group. Conversely, in a general population sample, a digitally delivered expressive writing exercise led to increased psychological distress [21]. Taken together, while the first study supports the notion that self-help internet interventions might be effective, the second study suggests that interventions proven effective in other settings might not in the current pandemic, implying that careful adaptation offering a variety of exercises and using multiple behavioral change techniques [22] may be needed.

Our research group has developed several cognitivebehavioral internet interventions over the last decade, serving as a construction kit for rapid new development. Building upon these interventions [23-25] and newly developed components, the intervention get.calm and move.on (GCMO) was created. GCMO is a cognitive-behavioral unguided self-help internet intervention with ten daily lessons. The aim of the current study was to evaluate the effectiveness of this novel program in reducing worry in the general population relative to internet-delivered psychoeducation.

\section{Materials and Methods}

Study Design

The current study was conducted as a two-arm, parallel, randomized controlled trial (RCT) at the Leuphana University of Lüneburg, Germany. The study was approved by the Ethics Committee of the University of Lüneburg, Germany and the protocol registered at the German Clinical Trials Registry (DRKS), the pri- 
mary German WHO registry (reference number: DRKS00021153). The study is reported in accordance to the CONSORT statement for nonpharmacological treatment trials.

\section{Participants}

Eligibility criteria were: (a) age 18 years or older, (b) no reported acute suicidal tendencies, and (c) no history of psychotic or dissociative symptoms. Participants were recruited mainly via reports in different media and social networks. No further inclusion or exclusion criteria were employed to facilitate access to the intervention for all interested individuals in the general population.

\section{Randomization and Masking}

Individuals were randomly assigned, using a computer-generated randomization list with a ratio of $1: 1$ and block size of two, to receive either GCMO, a cognitive-behavioral self-help internet intervention developed to reduce worries during the COVID-19 pandemic (intervention group), or internet-delivered psychoeducational advice on how to protect one's mental health (control group, mental health advice waiting group: MHA-W). Group allocation was conducted anonymously, with no personal contact between study personnel and participants. Blinding of participants was infeasible.

Participants in the GCMO group received immediate access to the intervention. Individuals in the MHA-W group were referred to a mental health advice website but granted access to the GCMO program after the 2-week follow-up assessment.

\section{Procedures}

Participants expressing their interest on the program's landing page (https://geton-training.de/get-calm-and-move-on/) were directed to a baseline questionnaire. Data collection was conducted online and occurred at baseline (T1) and after the intervention was completed ( 2 weeks after randomization, T2). Following the T2 assessment, the MHA-W group received access to the intervention. To assess the stability of the effects, we conducted a 2-month follow-up (T3) and a 6-month follow-up (T4) in both groups. Prior to completing the baseline questionnaire, participants were provided further information regarding the conditions of their participation, as well as details about the processing and handling of personal data. After individuals gave their written consent, inclusion criteria, demographic variables, and other outcome measures were assessed.

The GCMO program consists of ten daily sessions, each averaging 30-45 minutes in duration. The sessions include psychoeducational and cognitive-behavioral exercises that focus on maintaining daily structure, values-based behavioral activation, strategies to mentally disengage from worries, problem solving, relaxation, sleep hygiene, acceptance of unpleasant emotions, selfcare, and the activation of personal strengths and resources. The intervention was conceptualized and newly developed against the background of the pandemic in March 2020. We have made extensive adaptions to the COVID-19 pandemic (see online suppl. Table 2; see www.karger.com/doi/10.1159/000521302 for all online suppl. material). The intervention was designed to be an interactive self-help program, incorporating instructional videos, audio-guided imagination exercises, reading and writing sections, individualized feedback, and virtual companions (persona) to serve as role models for coping with problems related to the pandemic. The intervention did not include any guidance or human support. In order to enable replication [26], the components of the treatments are described in more detail in online supplementary Table 1.

The MHA-W group received official mental health recommendations on how to cope mentally with the pandemic from the German Society for Psychiatry and Psychotherapy, Psychosomatics and Neurology (see online suppl. Table 3). These recommendations (around 600 words and 3 minutes reading time) inform about the importance of a daily structure, social contact, acceptance of negative emotions and strengthening of positive emotions, and stimulus control to assimilate COVID-19-related news. The advice covers topics comparable to the WHO recommendations provided in "Coping with stress during the 2019-CoV outbreak," but in a more detailed manner. Those in the MHA-W group were informed that they would receive access to the same intervention that the GCMO group received after T2. The GCMO group did not receive the information that was provided to the MHA-W group. Both interventions advised individuals to contact physicians or psychotherapists and seek usual care if symptom deterioration occurred or if the low-threshold intervention was perceived to be unsatisfactory. The GCMO intervention made use of 19 different behavioral change techniques [22] as compared to two in the information given to the MHA-W group (see online suppl. Table 4). Therefore we expected that participants in the GCMO group were more likely to actually implement techniques and thus more likely for greater improvement on mental health outcomes.

\section{Outcomes}

The pre-specified primary outcome was level of worry, measured using the German version of the Penn State Worry Questionnaire (PSWQ) [27]. This scale consists of 15 items (e.g., "My worries overwhelmed me.") and has a total score that ranges from 0 to 90 . Scores $\geq 32.3$ can be regarded as moderate-high worry, scores $\geq 53.4$ as high worry [28] (for details see online suppl. Tables 5 and 6).

Secondary outcomes included generalized anxiety severity, measured using the seven-item version of the Generalized Anxiety Disorder (GAD-7) scale, which has a total score that ranges from 0 to 28 [29]. A score $\geq 10$ indicates moderate or severe levels of anxiety. Depression severity was measured with the eight-item version of the Patient Health Questionnaire (PHQ-8) depression scale [30], with a total score ranging from 0 to 24 . A total score $\geq 10$ indicates clinically significant levels of depression. Resiliency was measured with the Brief Resilience Scale (BRS) [31], consisting of six items, with a total score from 6 to 30 . Well-being was measured with the WHO-5 well-being index [32], consisting of five items, with a total score between 0 and 25. Self-efficacy was measured with the General Self-Efficacy Short Scale (GSES) [33], consisting of three items, with a total score ranging from 3 to 15 . To measure emotion regulation skills, the 27-item Emotion Regulations Skills Questionnaire (ERSQ) was used [34], for which total scores range from 0 to 108. Bivariate correlations of outcome variables to assess incremental validity [35] at T1 are depicted in online supplementary Table 7. To measure clients' satisfaction with the intervention, a validated version of the Client Satisfaction Questionnaire, adapted to the online context, was used (CSQ-I) [36]. The CSQ-I consists of eight items, with a total score ranging from 0 to 32 , with higher values corresponding to greater satisfaction. Demographic variables and COVID-19 infection status were collected at baseline. 
Statistical Analysis

For our sample size calculation, we followed DELTA guideline recommendations and specified the target difference between groups by considering both the practical importance of the effect and how realistic these effects are, based on meta-analytic evidence on the effects of online self-help interventions on anxiety for universal prevention [17]. Assuming that a difference of 3 points in the primary outcome is a meaningful and realistic effect, with a standard deviation of 10 points [27], we hypothesized a minimal clinically important difference between groups of $d=0.30$ at postintervention. Under these assumptions, a total (2-group) sample size of $N=352$ individuals was deemed necessary to detect the assumed effect with $80 \%$ power and a two-tailed significance level of $5 \%$.

Data analysis was performed using the statistical software program $\mathrm{R}$ (Version 1.3.959). A two-tailed significance level of $p \leq$ 0.05 was used for all inferential tests. Data were analyzed employing the intention-to-treat (ITT) principle. Missing data were estimated with multiple imputations, with 20 estimates calculated for each missing value. Imputed data sets were analyzed separately and the parameter estimates and hypothesis tests ultimately pooled. Existing data on the primary and secondary outcomes, as well as the grouping variable and sociodemographic variables, were used in the imputation model.

Considering analysis strategy, simulation studies demonstrated that analyses of covariance (ANCOVA) modeling would be more effective than either analysis of variance (ANOVA) or linear mixed modelling [37]. Accordingly, ANCOVA was employed to evaluate between-group differences at T2, including participant's baseline values as a covariate. To control for pandemic effects, each participant's and their significant other's COVID-19 infection status and the participant's time of registration for the intervention also were included as covariates. Between-group Cohen's $d$ values were calculated using pooled estimated marginal means and standard deviations.

For endpoints related to the two mental disorders of interest, using the GAD-7 anxiety and PHQ-8 depression scales, we calculated the number of participants reporting clinically relevant levels of symptoms, levels defined as scores equal to or above the clinical thresholds of 10 for the GAD-7 and 10 for the PHQ-8 at T2. Additionally, we calculated the number of participants reporting a $50 \%$ reduction in their symptoms of generalized anxiety and depression at T2. Numbers needed to treat (NNT) to achieve one clinical response in anxiety and in depression were then derived for both interventions.

To measure adverse effects, clinically significant deterioration was measured following a recently conducted individual participant meta-analysis on the side effects of internet interventions [26, 38]. Accordingly, deteriorations from $\mathrm{T} 1$ to $\mathrm{T} 2$ of $\geq 20.71$ points on the PSWQ, $\geq 5.36$ points on the PHQ-8, and $\geq 5.25$ on the GAD-7 were defined as adverse effects.

Exploratory moderation analysis was conducted to assess if baseline scores moderated the intervention's effect. Additionally, the Johnson-Neyman procedure was used to identify regions of significance.

The medium- and long-term effects were analyzed in a descriptive manner. Means, standard deviations, within-group change to baseline, and between-group Cohen's $d$ s were calculated.

iCBT versus Mental Health Advice to Reduce Worry during COVID-19 Pandemic

\section{Results}

The trial flow is depicted in Figure 1. The first participant enrolled on April 7, 2020 and the last participant on December 10, 2020. Of the 610 individuals who applied for participation, 372 filled out both the baseline and screening questionnaires. Of these, 352 were randomized to either the GCMO $(n=175)$ or MHA-W $(n=176)$ group. The last participant was randomized on December 11, 2020. The last participant out was on July 11, 2021. Due to an administrative error, one participant was randomized despite an age below the minimum 18 years required for study inclusion and was later excluded from the study.

Table 1 summarizes the sample's baseline characteristics. On average, $6.2(61.5 \%)$ of the ten GCMO sessions were completed ( $\mathrm{SD}=3.9$ ). Roughly $15 \%$ of the study participants reported actual infection with SARS-CoV-2 among close personal contacts, while $84 \%$ reported worrying about the possibility of infection among loved ones, $55 \%$ about themselves becoming infected, $43 \%$ about financial issues, and $31 \%$ about job insecurity caused by the pandemic. Further COVID-19-related worries at baseline and $\mathrm{T} 2$ are presented in online supplementary Table 8.

Overall, data were missing for the primary outcome for $12.5 \%$ of all participants at T2 (GCMO: 17.7\%; MHA$\mathrm{W}: 7.4 \%)$. At $\mathrm{T} 2$, individuals in the GCMO group reported significantly less worry than those in the MHA-W group $\left(F_{1,219}=12.9 ; p<0.001 ; \Delta=5.0\right.$ points; $d=0.38$; see Table 2). Moderation analysis revealed a significant group-by-baseline interaction for PSWQ baseline scores (est $=-0.29 ; \mathrm{SE}=0.08$; $t$ value $_{279.6}=-3.3 ; p<0.001$ ). Probing this effect with the Johnson-Neyman procedure revealed a baseline PSWQ score of 49.4 (95\% CI 48.9; 50.0), as the point of transition between a statistically nonsignificant and significant effect of GCMO, relative to MHA$\mathrm{W}$, on the PSWQ score at T2. This means that a significant between-group difference was identified for participants scoring 49.4 or greater on the baseline PSWQ score. This corresponds to $73.8 \%(n=259)$ of all study participants.

Per-protocol analysis, restricting analysis to participants who completed nine or more of the ten sessions (number of participants analyzed: GCMO: $N=75$; MHAW: $N=176)$ supported the results obtained with ITT analysis, but generated slightly larger effect sizes at T2 $\left(F_{1,241}=19.9 ; p<0.001 ; d=0.43\right)$.

Significant between-group differences also were identified for all secondary outcomes, except for self-efficacy. Specifically, generalized anxiety $(d=0.48)$ and depressive

Psychother Psychosom 2022;91:398-410 


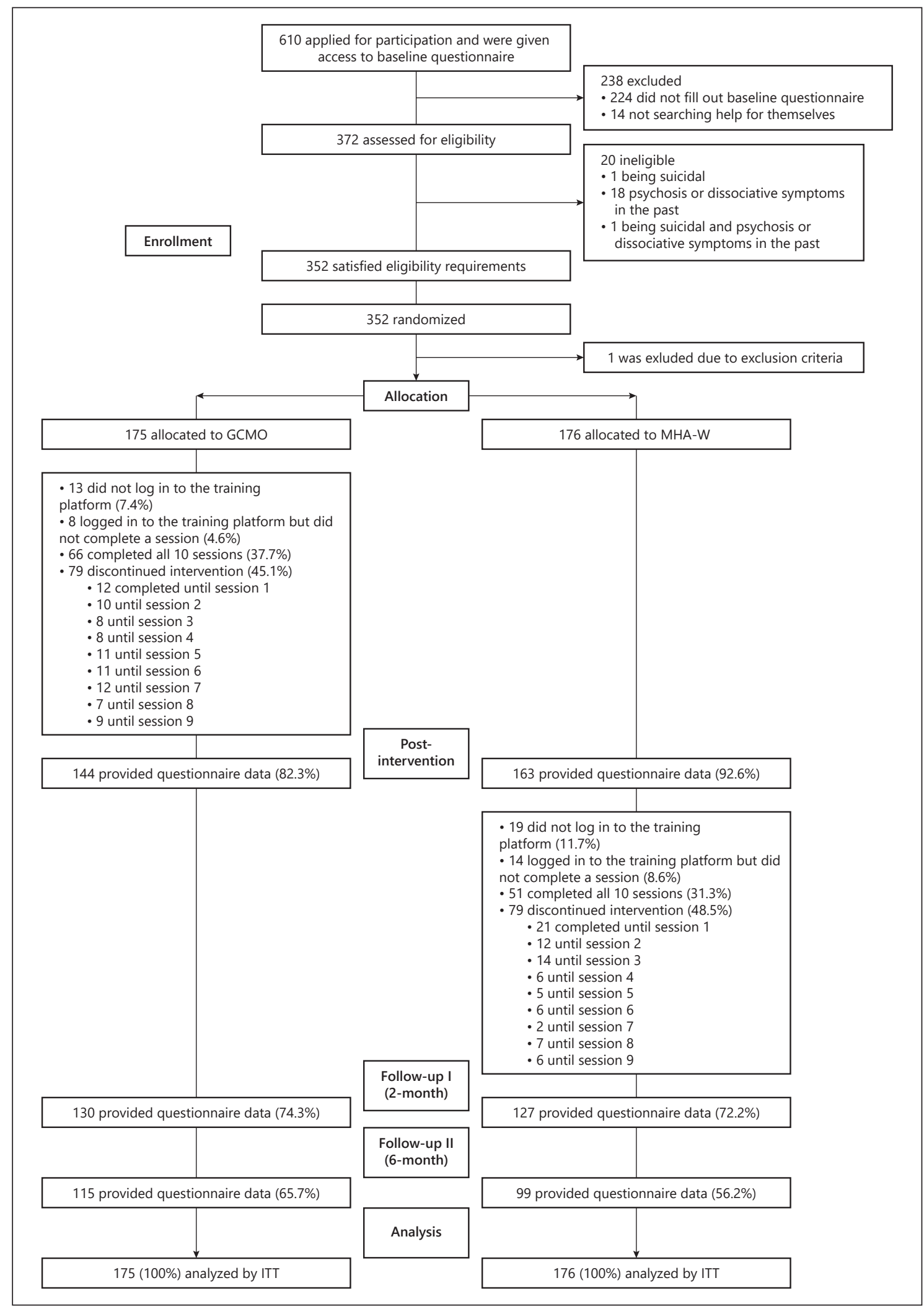

Fig. 1. Flow of participants. GCMO, get.calm and move.on, intervention group; MHA-W, mental health advice waiting group. 
Table 1. Baseline characteristics of the sample

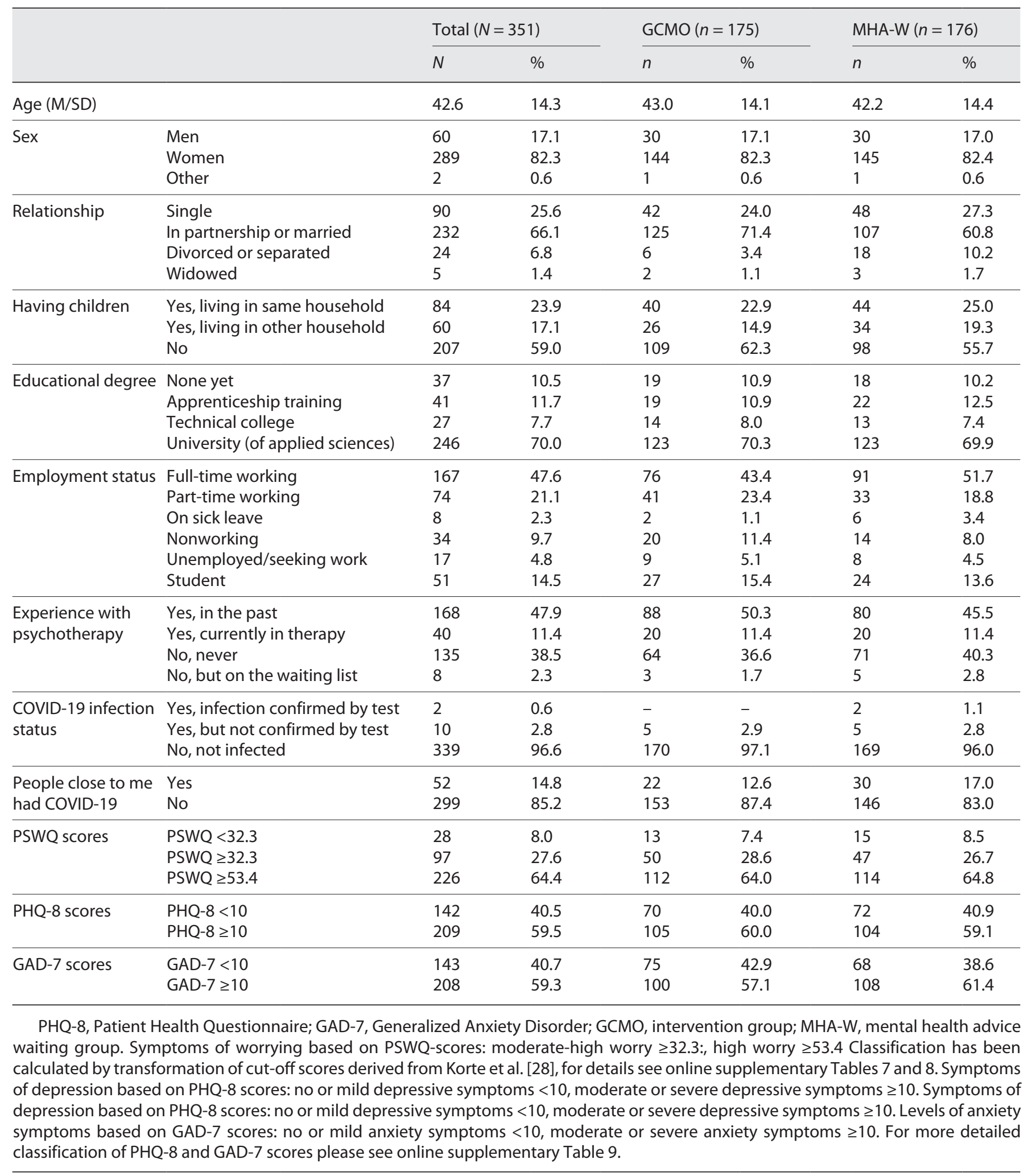

iCBT versus Mental Health Advice to Reduce Worry during COVID-19 Pandemic
Psychother Psychosom 2022;91:398-410 DOI: $10.1159 / 000521302$ 
Table 2. Means and standard deviations of the outcomes, results of ANCOVAs, and Cohen's ds for primary and secondary outcomes intention to treat sample

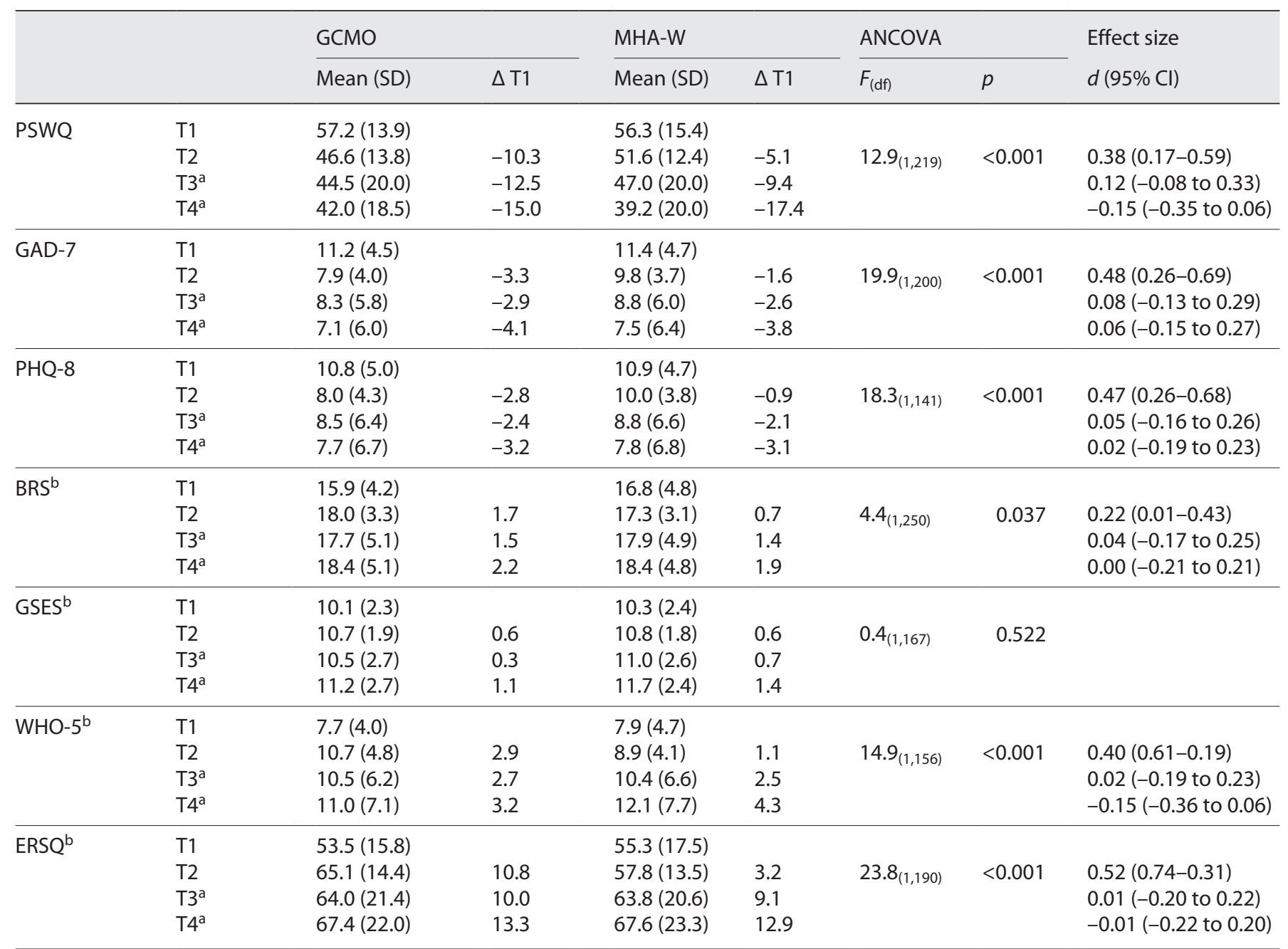

PSWQ, Penn State Worry Questionnaire; PHQ-8, Patient Health Questionnaire; GAD-7, Generalized Anxiety Disorder Scale; BRS, Brief Resilience Scale; GSES, General Self-Efficacy Short Scale; WHO-5, WHO-5 well-being index; ERSQ, Emotion Regulations Skills Questionnaire; GCMO, intervention group; MHA-W, mental health advice waiting group; T1, baseline; T2, post-intervention ( 2 weeks); T3, follow-up I (2 months); T4, follow-up II (6 months). Reported data is from the intention-to-treat sample. Therefore missing data was imputed by multiple imputations. Pooled estimated marginal means are reported. Scores for differences to baseline $(\Delta \mathrm{T} 1)$ refer to imputed, but unadjusted means at T1, T2 or T3. ${ }^{\mathrm{a}}$ The MHA-W group received the GCMO intervention after T2. For T3 and T4 no between-group differences were expected. ${ }^{\mathrm{b}}$ Higher values represent better outcomes.

symptoms $(d=0.47)$ were reduced more at T2 in the GCMO than MHA-W group. The distribution of participants into the different categories of symptom severity for worry, depression, and anxiety at all measurement points is depicted in online supplementary Tables 5 and 9 . Emotional regulation skills $(d=0.52)$, general well-being $(d=$ $0.40)$, and resiliency $(d=0.22)$ also were increased at T2 in the GCMO versus MHA-W group. At T2, 74.7\% of the participants assigned to the GCMO program reported no clinically relevant generalized anxiety symptoms $(n=$ $130.8)$, versus $47.4 \%(n=83.4)$ among their MHA-W counterparts, a difference that was highly significant $\left(\chi^{2}(1)=24.1 ; p<0.001\right)$. This corresponded to a NNT of 3.7 (95\% CI 2.7 ; 5.7) for one additional participant to experience a reduction in their GAD-7 score at T2 to nonclinically relevant status. At T2, 69.1\% of GCMO group subjects reported no clinically relevant symptoms of depression $(n=120.9)$, versus $45.3 \%(n=79.7)$ in the MHA- 
Fig. 2. Estimated means with $95 \%$ confidence intervals on the primary outcome measure (intention-to-treat; $N=351$ ). The MHA-W group received the GCMO intervention after T2. For T3 and T4 no between-group differences were expected. Penn State Worry Questionnaire (scale 0-90).

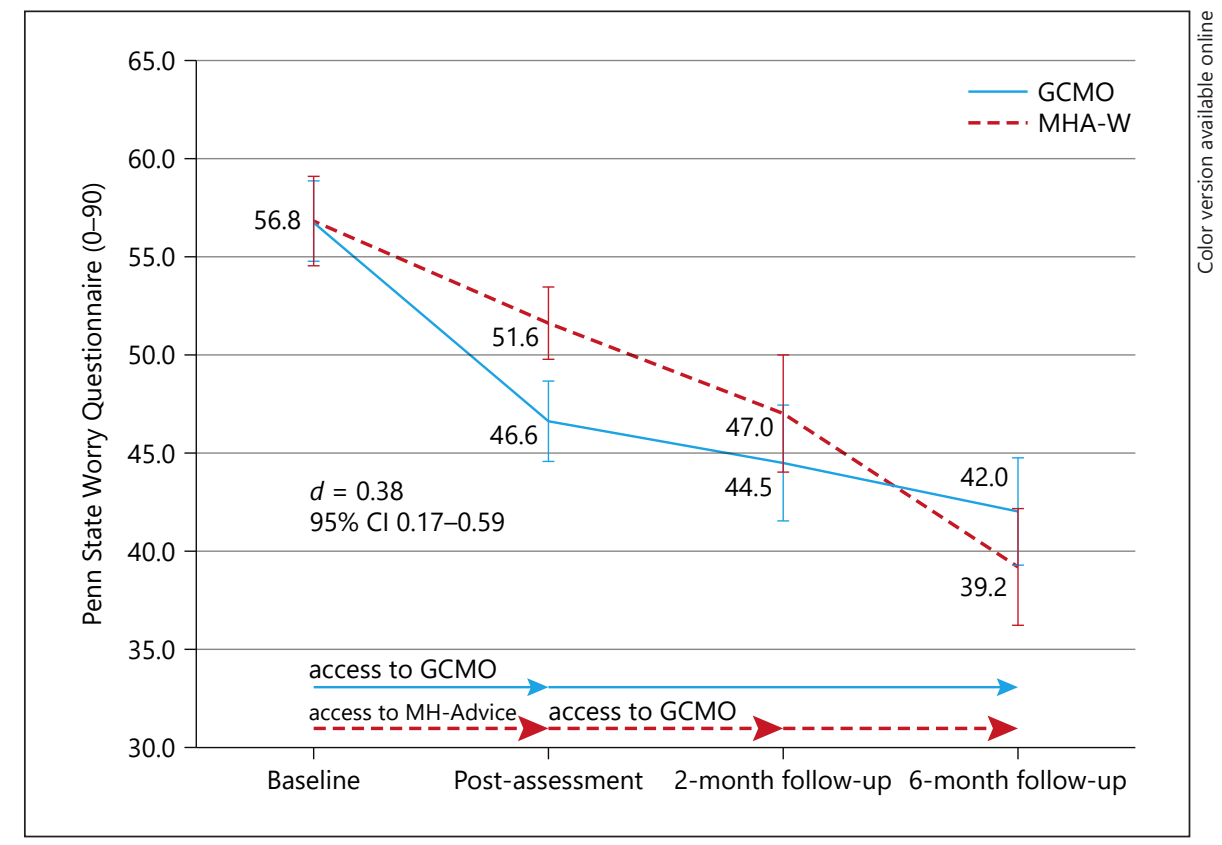

W group. This between-group difference in clinically relevant symptoms of depression also was significant $\left(\chi^{2}(1)\right.$ $=17.3 ; p<0.001)$. This corresponds to a NNT of $4.2(95 \%$ CI $3.0 ; 7.3)$ for one additional participant to achieve a reduction in their PHQ-8 score at T2 to non-clinically relevant status. Proportions of participants experiencing at least a $50 \%$ reduction in their symptoms relative to baseline are listed in online supplementary Table 10. Corresponding NNTs were 5.8 (95\% CI 3.9; 10.9) for generalized anxiety disorder and 4.7 (95\% CI 3.4; 7.5) for depression.

The client satisfaction questionnaire was completed by $76.0 \%$ of those allocated to the GCMO $(n=133)$. Overall satisfaction with the intervention was comparable to that reported for other, previously studied interventions $(\mathrm{M}=$ $26.6, \mathrm{SD}=5.3$, range $=8-32$ ) [36]. In an "overall, general sense," $56.4 \%(n=75)$ were very and $34.6 \%(n=46)$ mostly satisfied with the intervention they received, while 5.3\% $(n=7)$ were mildly and $3.8 \%(n=7)$ quite dissatisfied. The majority of the participants rated the sessions as rather or completely helpful with no clear preference for a particular session and about 85 to $95 \%$ reported to have actually conducted the exercises offered in the particular sessions (see online suppl. Tables 12 and 13). The majority (82.7\%) stated that they would have preferred a longer interval between sessions than the recommended one session per day. This preference was also expressed in the analysis of open text fields, in which participants were asked about possible improvements (see online suppl. Table 14). The majority $(67.7 \%)$ indicated that it would have been optimal for them to have one session every 3 to 4 days. With regard to implementation to daily life, participants most frequently (43\%) planned to continue using some form of gratitude exercise in the final session for their future (see online suppl. Table 15$)$. The majority $(85.7 \%)$ of the GCMO group at T2 claimed to have applied the exercises they had learned in the program in the past week, with $51.1 \%$ reporting using the exercises on occasional days, $21.1 \%$ on more than half of the days, and $13.5 \%$ almost every day.

A clinically significant deterioration at $\mathrm{T} 2$ in worrying was observed in $2(1.2 \%)$, in anxiety symptoms in $3(1.5 \%)$, and in depressive symptoms in 4 individuals $(2.5 \%)$ in the GCMO group, versus 1 (0.6\%), 4 (2.2\%), and 8 (4.7\%), respectively, in the MHA-W group. For respective numbers at T3 and T4, please see online supplementary Table 11.

With regards to mid- and long-term effects, Table 2 shows that the improvements made until T2 were maintained or extended until the 2-month (PSWQ $=44.5$; change -12.5 ) and 6-month follow-ups (PSWQ $=42.3$; change -14.7) for the GCMO group. After the MHA-W group had received the GCMO intervention following $\mathrm{T} 2$, the intensity of worry further decreased at 2-month (PSWQ $=47.0$; change -9.4 ) and 6-month follow-up (PSWQ $=40.7$; change -15.8$)$. Differences between groups at 2-month $(d=0.12)$ and 6-month follow-up ( $d$ $=-0.07)$ did not exceed the predefined minimal clinically important difference of $d=0.30$. 
Also see Figure 2 for the development of PSWQ scores over time. At T3 and T4 usage of the exercises did not differ between groups. $69.7 \%$ of the participants at T3 (59.1\% at T4) claimed to have applied the exercises they had learned in the program in the past week, with $56.2 \%$ $(48.3 \%$ at $\mathrm{T} 4)$ reporting using the exercises on occasional days, $8.0 \%$ (5.7\% at T4) on more than half of the days, and $5 \%(5.1 \%$ at $\mathrm{T} 4)$ almost every day.

\section{Discussion}

We found that the internet-based self-help intervention get.calm and move.on (GCMO), tailored to the COVID-19 pandemic, was effective at reducing worry to a larger extent compared to controls receiving officially endorsed internet-delivered advice to protect mental health during the pandemic and delayed access to GCMO. Additionally, we observed superior effectiveness of the GCMO program, in terms of reducing the symptoms of anxiety and depression, and the number of individuals who fell below the threshold of clinically significant symptoms for both mental conditions. Moreover, effects could be maintained up to 6 months. Other beneficial effects observed were enhanced emotional competency coping with stress, and increased resilience and well-being, though no effect on self-efficacy was apparent. Despite the program's rapid and lowbudget development, over $90 \%$ of the participants assigned to the GCMO program were satisfied with the intervention's quality.

To our knowledge, this study is the first to investigate an internet-based self-help intervention, accessible to the general population, developed to reduce worry during the COVID-19 pandemic. Results demonstrated rapid, clinically meaningful, and greater-than-expected relief in psychological distress in a most challenging situation. The study was designed from a public health and prevention perspective. As such, we focused primarily on worry reduction as an important risk factor and considered any psychopathology that might result from it [12], like depression and generalized anxiety disorder, as secondary outcomes, while the study reported by Wahlund and colleagues [20] focused on psychopathology. Given its different focus, the present study minimized inclusion criteria to mimic a universal prevention approach targeting the general population, contrary to Wahlund et al., who employed more restrictive eligibility criteria to assess a more indicated prevention strategy [20], excluding individuals with lower levels of wor- ry. While the effects were slightly higher for generalized anxiety disorder (GAD) symptoms in the latter study ( $d$ $=0.74$ vs. $d=0.48$ for GCMO), the effects were more similar for depressive symptoms $(d=0.38$ vs. $d=0.47$ GCMO). The latter study's greater effect on anxiety might be explained by both the higher level of GAD symptoms in the selected sample and, more importantly, the study's use of a non-active control group [39]. A strength of the present study was its inclusion of internet-delivered mental health advice in the waiting condition, covering topics similar to WHO recommendations, provided by the leading national association for psychiatry and psychotherapy. Descriptive data demonstrated a reduction of 5.1 points in worry from pre to post amongst controls in the MHA-W group. This is consistent with meta-analytic evidence suggesting beneficial effects of psychoeducation on depressive and stress-related symptoms [16], and might indicate the effectiveness of providing mental health advice from trusted and credible sources (i.e., national medical associations or the WHO) to the general population. Putting the effects of GCMO into the context of meta-analytic evidence for internet interventions targeting the general population in non-pandemic settings, the present effect sizes compare favorably for both anxiety $(d=$ $0.31)$ and depression $(d=0.25)$ [17]. Likewise, the antidepressant effects of GCMO were slightly greater than those calculated in an individual-participant meta-analysis of self-help and therapist-guided internet interventions designed to target subclinical depression $(\mathrm{g}=0.39)$ [18], and self-help interventions providing treatment to patients suffering from depression $(d=0.33)$ [40]. The number of individuals that must be treated to observe an additional clinically significant effect (NNT) of either a $50 \%$ reduction in depression symptoms or scoring below the clinical threshold for symptoms, also was superior for GCMO than the programs assessed in the aforementioned meta-analyses [18]. One reason for GCMO's superior beneficial effects might be its adherence to the recommendation for tailored information [41] and the variety of exercises and behavioral change techniques [22] offered. Accordingly, the intervention was adapted carefully to meet participants' needs in the current pandemic situation (see online suppl. Table 2). The importance of such adaptation was previously highlighted by Vukčević and colleagues [21], who found unexpected adverse effects in a stand-alone expressive writing exercise during the COVID-19 pandemic. They attributed these adverse effects to neglecting that COVID-19-related stressors were constantly present during the inter- 
vention and expressive writing usually focusses on past stressors. Additionally, more comprehensive digital interventions comprising various exercises like the program of Wahlund and colleagues [20] or GCMO might be more effective as they allow greater personalization compared to offering a single exercise [42]. In accordance with the literature, we defined adverse effects as clinically significant deteriorations in the symptoms of depression and generalized anxiety [38], and both were infrequently observed in either treatment condition. Even amongst the controls, the proportion of individuals with adverse effects did not exceed the rate of adverse effects observed among active intervention groups $(5.8 \%)$ in a recent individual-participant meta-analysis on the potential harmful effects of self-help internet interventions [38]. Notably, the same meta-analysis detected adverse effects in more than $9 \%$ of non-treated controls, highlighting the importance of providing at least a minimal level of intervention to participants in control groups.

Another strength of the present study is the assessment of mid- and long-term effects. The rapid and clinically meaningful relief after 2 weeks was maintained until the 2- and 6-month follow-up with a tendency of further improvement over time. Likewise, after the control group completed the GCMO intervention at T3 the effects were maintained at T4. It also appears noteworthy that nearly $60 \%$ of all participants at the 6-month follow-up reported to have used the strategies learned in the intervention in the last week. The stability of effects is in line with metaanalytic results for internet interventions for subthreshold depression [18] and may indicate that participants were able to successfully implement the strategies learned in this self-help intervention into everyday life. Our results suggest that gratitude exercises seem particularly popular with regard to implementation in daily life and may have contributed to the sustainable symptom reductions in worrying [25]. Since we designed the GCMO intervention to be accessible to anyone in the general population, it is itself of interest who elected to enroll in our study. First, the sociodemographic characteristics of our sample were similar to the sample population studied by Wahlund et al. [20]. Prior meta-analyses on internet interventions failed to consistently identify evidence to implicate subject gender, age, employment status, level of education, or past experience with psychotherapy as moderating variables $[18,40]$. Second, the medical (i.e., fear of infection) and non-medical (i.e., fear of financial worsening) COVID-related worries reported by our participants confirm results from observational studies re- porting psychological distress due to insecure life situations [7-9], and indicate that it was possible for us to access our intended target group with GCMO. Third, the high proportion of individuals with a history of psychotherapy and elevated symptoms of depression and anxiety indicates that the intervention reached a population with increased vulnerability. Finally, consistent with prior research involving non-clinical samples, higher levels of symptom severity at baseline predicted stronger effects of the internet interventions [18]. One advantage of the Johnson-Neyman technique we employed in our present analysis is that it specifies a threshold defining when an intervention has achieved a significant beneficial effect. Accordingly, individuals with PSWQ scores of 50 or more are likely to benefit more from the more intensive GCMO intervention than from mental health advice. This is an important finding, as it suggests that internet-delivered mental health advice is useful for those with lower levels of mental health complaints. However, public health strategies for mental health protection during a major pandemic should not be limited to recommendations, but be complemented with evidence-based self-help interventions for individuals with elevated levels of worry. Get. calm and move.on is, due to its self-help nature, a highly scalable internet-based intervention for the general population. It could be implemented at low costs as a preventive routine program, making an evidence-based intervention accessible for low- and middle-income countries, where resources are limited. As other public health interventions during the pandemic, such as vaccination or testing, are often disseminated by national or international health authorities, it seems worthwhile to augment these actions with public mental health measures, including interventions such as GCMO. However, recent metaanalyses suggest that internet-based interventions, when offered as part of routine care, show smaller effectiveness, which is partly due to reduced adherence $[43,44]$. To prevent the loss of adherence future research should therefore investigate whether additional motivational features [42] or the provision of human support increases the effectiveness in real-world settings.

Several limitations of our study must be considered. First, we did not include a third, non-treatment condition. Therefore, convincing conclusions about the effectiveness of the mental health advice that was offered are impossible, despite positive effects being observed from pre- to post-intervention. Based on existing evidence on psychoeducation and the perceived urgency for psychological support, we considered a non-treatment condition ethically inappropriate. Second, we conducted the 
post-intervention assessment very short-term, just 2 weeks after randomization. This was driven by ethical considerations and our aim to keep the waiting time for the control group as short as possible, since we expected that GCMO would most likely be of benefit to the participants. The majority of the participants stated that they were hardly able to adhere to the protocol of one session per day and preferred to practice the proposed strategies to cope with worry over a longer period of time. As a consequence of this short interval, the GCMO program's effects might have not become fully manifest, potentially underestimating the intervention's effectiveness. On the other hand, other researchers have found that effects develop rapidly within the first phase of an intervention [45]. Third, the levels of threat and hardship posed by the pandemic changed significantly over time, and moderating effects of the pandemic's various phases on GCMO's effectiveness cannot be excluded. However, statistically controlling for time and the infection status of both participants and their close contacts revealed no indication of such an effect. Fourth, replication is needed to further support these findings.

\section{Conclusion}

The current study's results suggest that a carefully adapted internet-based self-help intervention can be effective, even in extraordinary circumstances like a major pandemic. It sheds light on the heterogeneous findings on the effectiveness of internet-based self-help in general [40] and particularly on the previous mixed findings for interventions targeting mental health in the pandemic $[20,21]$. Interventions delivered over the internet are particularly helpful when physical distancing is mandated, a characteristic that may be especially important among those who fear face-to-face contact due to the heightened risk of infection. GCMO also provides fast and meaningful relief from worry, while simultaneously reducing the symptoms of generalized anxiety disorder and depression, thereby potentially preventing various forms of psychopathology from becoming compounded or chronic. It seems especially encouraging that participants were able to use the intervention in a way that led to stable reduction in worry up to 6 months after the intervention. Whilst mental health advice seems to be beneficial for those with average levels of worry, GCMO appears to be effective in those with more elevated levels of worry and is readily accessible to the general population at large.

\section{Acknowledgement}

Several researchers contributed to the development of prior internet interventions on stress management, detachment from repetitive negative thinking, insomnia, and gratitude. These interventions contributed to get.calm and move.on. We appreciate the work of (in alphabetical order): Matthias Berking, Pim Cuijpers, David Daniel Ebert, Henning Freund, Elena Heber, Hanne Horvath, and Heleen Riper. This research received no grants from any external source. We thank Lea Liebchen for her support in the development of the virtual companions for the intervention and Jannik Porath for his support with study administration.

We acknowledge the engagement of the German Association for Psychiatry, Psychotherapy and Psychosomatics (DGPPN) in providing free mental health recommendations to the public.

\section{Statement of Ethics}

The authors assert that all procedures contributing to this work comply with the ethical standards of the relevant national and institutional committees on human experimentation and with the Helsinki Declaration of 1975 , as revised in 2008 . This study protocol was reviewed and approved by the Ethics Committee of the University of Lüneburg, Germany, approval number [EB-Antrag_202003_12_ Lehr_getcalm]. Written informed consent was obtained from participants to participate in the study prior to baseline assessment.

\section{Conflict of Interest Statement}

H.H., D.L., and L.B. declare no competing interests.

\section{Funding Sources}

Leuphana University of Lüneburg informed about the study in a press release and had no further role in study design; data collection, analysis, or interpretation; or writing of the report. There was no external funding source for this study.

\section{Author Contributions}

H.H. initiated the study, developed and programmed the intervention, conceptualized the study, contributed to the literature search, was responsible for designing and programming the study and instruments, was the trial manager, prepared, analyzed, and interpreted the data, wrote the first draft, and critically reviewed and edited the manuscript. D.L. developed and programmed the intervention, conceptualized the study, contributed to the literature search, was responsible for designing the study and its instruments, supervised the trial manager, communicated with the ethics committee, interpreted the data, wrote the first draft, and critically reviewed and edited the manuscript. L.B. conducted setup and maintenance of the technical platform, contributed to the literature search, accessed, verified, analyzed, and interpreted the data, and critically reviewed the manuscript. 


\section{Data Availability Statement}

Individual participant data that underlie the results reported in this article, after de-identification (text, tables, figures, and online suppl. material) will be made available upon request with researchers who provide a methodologically sound proposal.

\section{Intervention Sharing Statement}

Researchers, public health agencies, and other non-profit organizations that intend to implement the intervention are encouraged to contact Dirk Lehr (lehr@leuphana.de) for permission to provide GCMO in any country worldwide on a free-of-charge basis.

\section{References}

1 Brooks SK, Webster RK, Smith LE, Woodland L, Wessely S, Greenberg N, et al. The psychological impact of quarantine and how to reduce it: rapid review of the evidence. Lancet. 2020 Mar;395(10227):912-20.

2 Kunzler AM, Röthke N, Günthner L, StoffersWinterling J, Tüscher $\mathrm{O}$, Coenen $\mathrm{M}$, et al. Mental burden and its risk and protective factors during the early phase of the SARSCoV-2 pandemic: systematic review and meta-analyses. Glob Health. 2021 Dec;17(1):34.

3 Pan K-Y, Kok AAL, Eikelenboom M, Horsfall M, Jörg F, Luteijn RA, et al. The mental health impact of the COVID-19 pandemic on people with and without depressive, anxiety, or obsessive-compulsive disorders: a longitudinal study of three Dutch case-control cohorts. Lancet Psychiatry. 2021 Feb;8(2):121-9.

4 Pierce M, Hope H, Ford T, Hatch S, Hotopf $\mathrm{M}$, John A, et al. Mental health before and during the COVID-19 pandemic: a longitudinal probability sample survey of the UK population. Lancet Psychiatry. 2020 Oct;7(10): 883-92.

5 Cosci F, Guidi J. The role of illness behavior in the COVID-19 pandemic. Psychother Psychosom. 2021;90(3):156-9.

6 Soklaridis S, Lin E, Lalani Y, Rodak T, Sockalingam S. Mental health interventions and supports during COVID- 19 and other medical pandemics: a rapid systematic review of the evidence. Gen Hosp Psychiatry. 2020 Sep; 66:133-46.

7 Roy D, Tripathy S, Kar SK, Sharma N, Verma SK, Kaushal V. Study of knowledge, attitude, anxiety \& perceived mental healthcare need in Indian population during COVID-19 pandemic. Asian J Psychiatr. 2020 Jun;51:102083.

8 Taylor S, Landry CA, Paluszek MM, Rachor GS, Asmundson GJG. Worry, avoidance, and coping during the COVID-19 pandemic: a comprehensive network analysis. J Anxiety Disord. 2020 Dec;76:102327.

9 Tull MT, Edmonds KA, Scamaldo KM, Richmond JR, Rose JP, Gratz KL. Psychological outcomes associated with stay-at-home orders and the perceived impact of COVID-19 on daily life. Psychiatry Res. 2020 Jul;289: 113098.

10 Borkovec TD, Robinson E, Pruzinsky T, DePree JA. Preliminary exploration of worry: some characteristics and processes. Behav Res Ther. 1983;21(1):9-16.
11 Ehring T, Zetsche U, Weidacker K, Wahl K, Schönfeld S, Ehlers A. The Perseverative Thinking Questionnaire (PTQ): validation of a content-independent measure of repetitive negative thinking. J Behav Ther Exp Psychiatry. 2011 Jun;42(2):225-32.

12 Watkins ER. Constructive and unconstructive repetitive thought. Psychol Bull. 2008; 134(2):163-206.

13 Drost J, van der Does W, van Hemert AM, Penninx BW, Spinhoven P. Repetitive negative thinking as a transdiagnostic factor in depression and anxiety: a conceptual replication. Behav Res Ther. 2014 Dec;63:177-83.

14 Andersson G, Titov N. Advantages and limitations of Internet-based interventions for common mental disorders. World Psychiatry. 2014 Feb;13(1):4-11.

15 World Health Organization. Coping with stress during the 2019 -nCoV outbreak [Internet]. 2020 [cited 2020 Mar 23]. Available from: https: //www.who.int/docs/defaultsource/coronaviruse/coping-with-stress. pdf?sfvrsn=9845bc3a_2.

16 Donker T, Griffiths KM, Cuijpers P, Christensen H. Psychoeducation for depression, anxiety and psychological distress: a metaanalysis. BMC Med. 2009 Dec;7(1):79.

17 Deady M, Choi I, Calvo RA, Glozier N, Christensen $\mathrm{H}$, Harvey SB. eHealth interventions for the prevention of depression and anxiety in the general population: a systematic review and meta-analysis. BMC Psychiatry. 2017 Dec;17(1):310.

18 Reins JA, Buntrock C, Zimmermann J, Grund S, Harrer M, Lehr D, et al. Efficacy and moderators of internet-based interventions in adults with subthreshold depression: an individual participant data meta-analysis of randomized controlled trials. Psychother Psychosom. 2021;90(2):94-106.

19 Wright JH, Caudill R. Remote treatment delivery in response to the COVID-19 pandemic. Psychother Psychosom. 2020;89(3):130-2.

20 Wahlund T, Mataix-Cols D, Olofsdotter Lauri K, de Schipper E, Ljótsson B, Aspvall K, et al. Brief online cognitive behavioural intervention for dysfunctional worry related to the COVID-19 pandemic: a randomised controlled trial. Psychother Psychosom. 2021; 90(3):191-9.
21 Vukčević Marković M, Bjekić J, Priebe S. Effectiveness of expressive writing in the reduction of psychological distress during the COVID-19 pandemic: a randomized controlled trial. Front Psychol. 2020 Nov; 11: 587282.

22 Michie S, Wood CE, Johnston M, Abraham C, Francis JJ, Hardeman W. Behaviour change techniques: the development and evaluation of a taxonomic method for reporting and describing behaviour change interventions (a suite of five studies involving consensus methods, randomised controlled trials and analysis of qualitative data). Health Technol Assess. 2015 Nov; 19(99):1-188.

23 Heber E, Lehr D, Ebert DD, Berking M, Riper $\mathrm{H}$. Web-based and mobile stress management intervention for employees: a randomized controlled trial. J Med Internet Res. 2016 Jan; 18(1):e21.

24 Thiart H, Lehr D, Ebert DD, Berking M, Riper H. Log in and breathe out: internet-based recovery training for sleepless employees with work-related strain - results of a randomized controlled trial. Scand J Work Environ Health. 2015 Mar;41(2):164-74.

25 Heckendorf H, Lehr D, Ebert DD, Freund H. Efficacy of an internet and app-based gratitude intervention in reducing repetitive negative thinking and mechanisms of change in the intervention's effect on anxiety and depression: results from a randomized controlled trial. Behav Res Ther. 2019 Aug; 119: 103415 .

26 Guidi J, Brakemeier E-L, Bockting CLH, Cosci F, Cuijpers P, Jarrett RB, et al. Methodological recommendations for trials of psychological interventions. Psychother Psychosom. 2018;87(5):276-84.

27 Stöber J, Bittencourt J. Weekly assessment of worry: an adaptation of the Penn State Worry Questionnaire for monitoring changes during treatment. Behav Res Ther. 1998 Jun; 36(6):645-56.

28 Korte KJ, Allan NP, Schmidt NB. Factor mixture modeling of the Penn State Worry Questionnaire: evidence for distinct classes of worry. J Anxiety Disord. 2016 Jan;37:40-7.

29 Spitzer RL, Kroenke K, Williams JB, Löwe B. A brief measure for assessing generalized anxiety disorder: the GAD-7. Arch Intern Med. 2006 May;166(10):1092. 
30 Kroenke K, Strine TW, Spitzer RL, Williams JB, Berry JT, Mokdad AH. The PHQ- 8 as a measure of current depression in the general population. J Affect Disord. 2009 Apr;114(13):163-73.

31 Smith BW, Dalen J, Wiggins K, Tooley E, Christopher P, Bernard J. The brief resilience scale: assessing the ability to bounce back. Int J Behav Med. 2008 Sep;15(3):194-200.

32 Brähler E, Mühlan H, Albani C, Schmidt S. Teststatistische Prüfung und Normierung der deutschen Versionen des EUROHIS-QOL Lebensqualität-Index und des WHO-5 Wohlbefindens-Index. Diagnostica. 2007 Apr; 53(2):83-96.

33 Beierlein C, Kovaleva A, Kemper CJ, Rammstedt B. Ein Messinstrument zur Erfassung subjektiver Kompetenzerwartungen: Allgemeine Selbstwirksamkeit Kurzskala (ASKU). GESIS-Work Pap 201217 Mannh GESIS. Köln: Leibniz-Inst Für Sozialwissenschaften; 2012.

34 Berking M, Znoj H. Entwicklung und Validierung eines Fragebogens zur standardisierten Selbsteinschätzung emotionaler Kompetenzen (SEK-27). Z Für Psychiatr Psychol Psychother. 2008 Jan;56(2):141-53.

35 Carrozzino D, Patierno C, Guidi J, Berrocal Montiel C, Cao J, Charlson ME, et al. Clini- metric criteria for patient-reported outcome measures. Psychother Psychosom. 2021; 90(4):222-32.

36 Boß L, Lehr D, Reis D, Vis C, Riper H, Berking $\mathrm{M}$, et al. Reliability and validity of assessing user satisfaction with web-based health interventions. J Med Internet Res. 2016 Aug;18(8): e234.

37 O Connell NS, Dai L, Jiang Y, Speiser JL, Ward R, Wei W, et al. Methods for analysis of pre-post data in clinical research: a comparison of five common methods. J Biom Biostat. 2017;8(1):1-8.

38 Karyotaki E, Kemmeren L, Riper H, Twisk J, Hoogendoorn A, Kleiboer A, et al. Is selfguided internet-based cognitive behavioural therapy (iCBT) harmful? An individual participant data meta-analysis. Psychol Med. 2018 Nov;48(15):2456-66.

39 Mohr DC, Spring B, Freedland KE, Beckner V, Arean P, Hollon SD, et al. The selection and design of control conditions for randomized controlled trials of psychological interventions. Psychother Psychosom. 2009;78(5): 275-84.

40 Karyotaki E, Riper H, Twisk J, Hoogendoorn A, Kleiboer A, Mira A, et al. Efficacy of selfguided internet-based cognitive behavioral therapy in the treatment of depressive symp- toms: a meta-analysis of individual participant data. JAMA Psychiatry. 2017 Apr;74(4):351.

41 Kreuter MW, Wray RJ. Tailored and targeted health communication: strategies for enhancing information relevance. Am J Health Behav. 2003 Nov;27 Suppl 3(1):S227-32.

42 Wei Y, Zheng P, Deng H, Wang X, Li X, Fu H. Design features for improving mobile health intervention user engagement: systematic review and thematic analysis. J Med Internet Res. 2020 Dec;22(12):e21687.

43 Romijn G, Batelaan N, Kok R, Koning J, van Balkom A, Titov N, et al. Internet-delivered cognitive behavioral therapy for anxiety disorders in open community versus clinical service recruitment: meta-analysis. J Med Internet Res. 2019 Apr;21(4):e11706.

44 Etzelmueller A, Vis C, Karyotaki E, Baumeister $\mathrm{H}$, Titov N, Berking M, et al. Effects of internet-based cognitive behavioral therapy in routine care for adults in treatment for depression and anxiety: systematic review and meta-analysis. J Med Internet Res. 2020 Aug. 22(8):e18100.

45 Owen J, Adelson J, Budge S, Wampold B, Kopta M, Minami T, et al. Trajectories of change in psychotherapy: international center for clinical excellence. J Clin Psychol. 2015 Sep;71(9):817-27. 